\title{
UPLC-MS/MS Determination of Twelve Ginsenosides in Shenfu Tang and Dushen Tang
}

\author{
Dawai Yang $\left(D,{ }^{1}\right.$ Xiaofang Yang, ${ }^{2}$ Han Yan, ${ }^{2}$ Bin Fan, ${ }^{2}$ Jingang Dai, ${ }^{2}$ Jun Song, \\ Yan Lei $\left({ }^{2},{ }^{2}\right.$ and Na Guo $\varpi^{2}$ \\ ${ }^{1}$ Zhong Yuan Academy of Biological Medicine, Liaocheng People's Hospital, Liaocheng University, Liaocheng 252000, China \\ ${ }^{2}$ Experimental Research Center, China Academy of Chinese Medical Sciences, Beijing 100700, China
}

Correspondence should be addressed to Yan Lei; 13651217893@163.com and Na Guo; guona5246@126.com

Dawai Yang and Xiaofang Yang contributed equally to this work.

Received 2 March 2019; Revised 28 May 2019; Accepted 17 June 2019; Published 11 July 2019

Academic Editor: Pavel Nesterenko

Copyright (C) 2019 Dawai Yang et al. This is an open access article distributed under the Creative Commons Attribution License, which permits unrestricted use, distribution, and reproduction in any medium, provided the original work is properly cited.

\begin{abstract}
Shenfu Tang and Dushen Tang (one of the composite medicines for Shenfu Tang) are widely used Traditional Chinese herbal formulations and ginsenosides are their main bioactive components. However, there are rare studies about simultaneous analysis of ginsenosides in Shenfu Tang and Dushen Tang. In order to identify ginsenosides in Shenfu Tang and Dushen Tang and to explore law of compatibility of medicines in the decoction, a method for simultaneous determination of twelve ginsenosides in Shenfu Tang and Dushen Tang was developed by ultraresolution liquid chromatography coupled with tandem mass spectrometry (UPLC-MS/MS). The method showed satisfactory linearity $(r>0.9915)$, repeatability (RSD $<9.58 \%)$, intra- and interday precisions (RSD $<11.90 \%$ ), and high yields of recovery (92.26-113.20\%) for twelve major constituents, namely, ginsenosides-Rb1, Rb2, Rb3, Rc, Rd, Rg1, Re, Rf, Rg2, Rg3, Rh1, and F2. Furthermore, the concentration of twelve ginsenosides in Dushen Tang and Shenfu Tang was also simultaneously analyzed. Most of ginsenosides except Rgl and Rbl showed higher contents in Shenfu Tang compared to Dushen Tang. The compatibility of the formula had the effect of promoting or inhibiting the dissolution of some major components. The present research provided a reliable evidence for the illustration of chemical basis and compatibility regularity of Shenfu Tang. This study demonstrated the utility of the developed method for assessment of the quantity of the major constituents in Dushen Tang and Shenfu Tang.
\end{abstract}

\section{Introduction}

Traditional Chinese herbal formulation has been widely used in the clinic for its well-proven efficacy with few side effects $[1,2]$. Decoction is the traditional prescription of traditional Chinese medicines (TCM). One single herb or several kinds of herbs combined are boiled in water to make the decoction based on TCM theory [3]. Shenfu Tang, which was documented originally in 1465 , is a famous TCM decoction with 3:2 ratio of Radix Ginseng and Fuzi (Radix Aconiti lateralis praeparata). It has been widely used to treat various diseases with signs of Yangqi decline or Yang exhaustion and especially for cardiovascular diseases [4-6]. Dushen Tang, which was traced back to 1600 years ago, is the decoction of the root of single Panax ginseng [3] and one of the composite medicines for Shenfu Tang. Ginseng is commonly used medicinal plant in both East Asia and the West and has been used as a tonic and a panacea that enhance physical performance and increase resistance to stress and aging $[7,8]$.

The main bioactive components of Dushen Tang and Shenfu Tang are ginsenosides since their major components are ginseng according to their formula. Ginsenosides have multiple pharmacological activities, including antioxidant, anti-inflammatory, antifatigue, and immunityimproving properties [9]. Therefore, it is necessary to investigate phytochemistry, metabolism, and quantity of ginsenosides in Dushen Tang and Shenfu Tang using modern technologies. To date, there were about 150 ginsenosides that were identified in the previous studies [10].

Many techniques including HPLC [11-13], GC-MS [14, 15], and LC-MS [16-20] were used to analyze ginsenosides. 


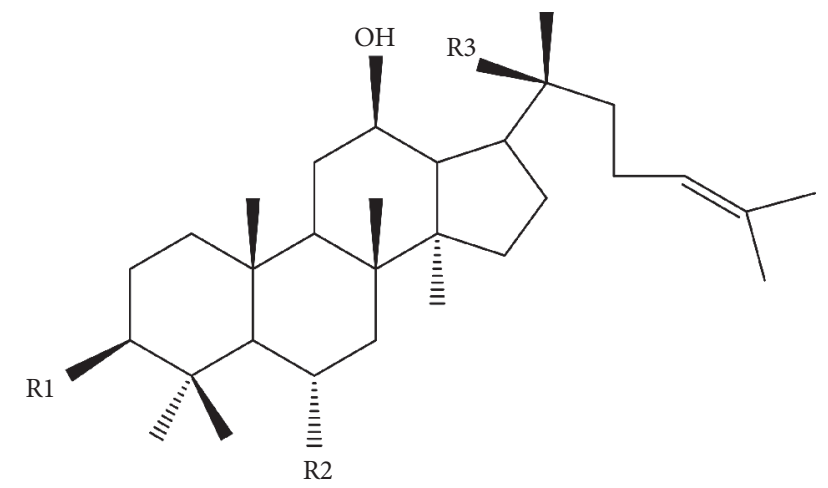

\begin{tabular}{|c|c|c|c|}
\hline Compound & $\mathrm{R} 1$ & $\mathrm{R} 2$ & R3 \\
\hline \multicolumn{4}{|c|}{ PPD-type ginsenosides } \\
\hline Ginsenoside-Rb1 & -O-Glc2-1 Glc & $-\mathrm{H}$ & -O-Glc6- \\
\hline Ginsenoside- $\mathrm{Rb} 2$ & -O-Glc2-1 Glc & $-\mathrm{H}$ & -O-Glc6- \\
\hline Ginsenoside-Rc & -O-Glc2-1 Glc & $-\mathrm{H}$ & -O-Glc6- \\
\hline Ginsenoside-Rd & -O-Glc2-1 Glc & $-\mathrm{H}$ & -O-Glc \\
\hline Ginsenoside-Rb3 & -O-Glc2-1 Glc & $-\mathrm{H}$ & -O-Glc6- \\
\hline Ginsenoside-Rg3 & -O-Glc2-1 Glc & $-\mathrm{CH} 3$ & $-\mathrm{OH}$ \\
\hline Ginsenoside-F2 & -O-Glc & $-\mathrm{H}$ & -O-Glc \\
\hline \multicolumn{4}{|c|}{ PPT-type ginsenosides } \\
\hline Ginsenoside-Re & $-\mathrm{OH}$ & -O-Glc2-1 Rha & -O-Glc \\
\hline Ginsenoside-Rg1 & $-\mathrm{OH}$ & $-\mathrm{O}-\mathrm{Glc}$ & -O-Glc \\
\hline Ginsenoside-Rf & $-\mathrm{OH}$ & -O-Glc2-1 Glc & $-\mathrm{OH}$ \\
\hline Ginsenoside-Rh1 & $-\mathrm{OH}$ & $-\mathrm{O}-\mathrm{Glc}$ & $-\mathrm{OH}$ \\
\hline Ginsenoside-Rg2 & $-\mathrm{OH}$ & -O-Glc2-1 Rha & $-\mathrm{OH}$ \\
\hline
\end{tabular}

FIGURE 1: Chemical structures of ginsenosides.

Among them, the LC-MS and tandem mass spectrometry (MS/MS) techniques have been widely used for analysis of ginsenosides, because of their high dynamic range of detection, high sensitivity, and specificity [3, 21]. Many works have been carried out for the identification, quantification, and quality control of ginsenosides in raw plant materials, extracts, and marketed products [22-24]. Ginsenosides-Rb1, Rb2, Rc, Rd, Rg1, Re, and Rf (Figure 1) have been identified in Dushen Tang and Shenfu Tang in the previous studies $[19,25]$. But simultaneous comparatively determination of twelve ginsenosides in Dushen Tang and Shenfu Tang has not been studied until now. Therefore, in order to further find out the prescriptions of traditional Chinese medicine, we confirmed major compounds in Dushen Tang and Shenfu Tang and determined them simultaneously by using UPLCMS/MS

The aim of the present study was to develop a direct and rapid LC-MS/MS method to simultaneously quantify twelve ginsenosides in Shenfu Tang and one of its composite medicines, Dushen Tang, namely, ginsenosides$\mathrm{Rb}_{1}, \mathrm{Rb}_{2}, \mathrm{Rb} 3, \mathrm{Rc}, \mathrm{Rd}, \mathrm{Rg}_{1}$, Re, Rf, Rg2, Rg3, Rh1, and F2 (Figure 1). In addition, the concentration of twelve ginsenosides in Dushen Tang and Shenfu Tang was also determined.

\section{Materials and Methods}

2.1. Chemicals, Standards, and Samples. MS-grade acetonitrile and formic acid were purchased from Fisher Scientific (USA). All other chemicals and solvents were of an analytical grade. Ultrapure water (18.2AM $\Omega$ ) was prepared with a MilliQ water purification system (Millipore, Bedford, MA, USA). The reference standards of ginsenosides- $R b_{1}, R_{2}, R b 3, R c$, $\mathrm{Rd}, \mathrm{Rg}_{1}$, Re, Rf, Rg2, Rg3, Rh1, and F2 were purchased from the National Institute for Control of Pharmaceutical and Biological Products (Beijing, China). The structures of these compounds are listed in Figure 1. The purity of the standards was no less than $98 \%$. The commercial white ginseng samples were purchased from Jilin Shengyuan Changbai Mountain Pharmaceutical Co., Ltd. in China. The processed aconite root (Radix Aconiti lateralis praeparata) was purchased from Tong-Ren-Tang Pharmaceutical store (Beijing, China) and authenticated by Professor Xirong, HE, Institute of Traditional Chinese Medicine, China Academy of Chinese Medical Sciences. 


\subsection{Sample Preparation}

2.2.1. Reference Standards Solutions. Stock solutions: certain amounts of ginsenoside- $\mathrm{Rb}_{1}, \mathrm{Rb}_{2}, \mathrm{Rb} 3, \mathrm{Rc}, \mathrm{Rd}, \mathrm{Rg}_{1}, \mathrm{Re}$, $\mathrm{Rf}, \mathrm{Rg} 2, \mathrm{Rg} 3$, Rh1, and F2 were dissolved in methanol, respectively, to get reference standards solutions $(1.0 \mathrm{mg} / \mathrm{mL})$ and were stored below $4^{\circ} \mathrm{C}$.

2.2.2. Extracts of Shenfu Tang and Dushen Tang. Shenfu Tang is a TCM prescription with a 3:2 ratio of Radix Ginseng and Fuzi (Radix Aconiti lateralis praeparata). Dried and pulverised white ginseng (1200 g) and the processed aconite root $(800 \mathrm{~g})$ were ground and then refluxed three times with $20 \mathrm{~L}$ of water for $60 \mathrm{~min}$ at $100^{\circ} \mathrm{C}$ based on our published papers $[19,25]$. After cooling, the extracting solutions were filtered and condensed under decompression and finally were freeze-dried. The extraction rate is $31.93 \%$.

Dried and pulverised white ginseng $(1000 \mathrm{~g})$ were ground and then refluxed three times with $10 \mathrm{~L}$ of water for 60 min at $100^{\circ} \mathrm{C}$. After cooling and filtering, the solutions were condensed under decompression and were finally freezedried. The extraction rate is $42.4 \%$.

The decoction extracts were dissolved, respectively, in a certain amount of water to a concentration $10 \mathrm{mg} / \mathrm{mL}$. 0.5 $\mathrm{mL}$ of the solutions was precipitated, respectively, with $4 \mathrm{~mL}$ ethanol allowed to sit for $24 \mathrm{~h}$ at $4^{\circ} \mathrm{C}$. The solutions were centrifuged at $4500 \mathrm{rpm}$ for $10 \mathrm{~min}$. After that, the solutions were diluted 50 times and centrifuged at $4000 \mathrm{r} / \mathrm{min}$ for 5 minutes.

2.3. Method Validation. An external calibration method was used for the quantitative analysis. The linearity calibration curves were constructed by six different concentrations of twelve ginsenosides. Each concentration was analyzed in triplicate and then the calibration curves were constructed by plotting the peak areas versus the concentrations of each analyte. The limit of detection (LOD) and limit of quantification (LOQ) were measured with the signal-tonoise ratio of 3 and 10, respectively, as criteria. The intraday precision was performed by analysis of the standard solution at six times within 1 day, while the interday precision was determined by repeated analysis of the sample for consecutive 3 days. For repeatability test, six independent sample solutions were prepared in the procedures noted. For stability test, the sample solutions set for three hours to 24 hours were analyzed. The recovery of this method was achieved using the standard addition method. The average recoveries were determined by the following formula: Recovery (\%) $=($ Observed amount-Original amount $) /$ Spiked amount $\times 100$ $\%$, RSD $(\%)=(\mathrm{SD} /$ mean $) \times 100 \%$.

2.4. UPLC-MS Conditions. A Waters ACQUITY UPLC system (Milford, MA, USA), which was equipped with a Waters (Milford, MA, USA) Xevo TQ-S triple quadrupole mass spectrometer and operated in positive ion mode (data analysis software MassLynx ${ }^{\mathrm{TM}}$ V4.1), was used for simultaneous determination of twelve ginsenosides in Shenfu Tang. The separation was performed on ACQUITY UPLC BEH C18 $(100 \mathrm{~mm} \times 2.1 \mathrm{~mm}, 1.8 \mu \mathrm{m})$. The mobile phase consisted of
(A) water containing $0.1 \%$ formic acid and (B) acetonitrile containing $0.1 \%$ formic acid. The linear gradient conditions were as follows: $0.0-1.5 \mathrm{~min}, 100.0-80.0 \% \mathrm{~B} ; 1.5-3.0 \mathrm{~min}, 80.0$ 73.0 \% ; 3.0-5.0 min, 73.0-73.0 \%; 5.0-5.5 min, 73.0-72.0 \%; 5.5$12.0 \mathrm{~min}, 72.0-70.0 \%$; $12.0-15.0 \mathrm{~min}, 70.0-69.0 \%$; $15.0-18.5$ $\min , 69.0-50.0 \%$; $18.5-21.5 \mathrm{~min}, 50.0-40.0 \%$; 18.5-21.5 min, 50.0-40.0 \%; and 21.5-22.0 $\mathrm{min}, 40.0-0.0 \%$. The flow rate was $0.30 \mathrm{ml} / \mathrm{min}$. The column temperature was $45^{\circ} \mathrm{C}$.

The conditions of MS analysis were as follows: the desolvation gas was set to $600 \mathrm{~L} / \mathrm{h}$ at temperature of $400^{\circ} \mathrm{C}$; the cone gas was set to $150 \mathrm{~L} / \mathrm{h}$, respectively. The capillary voltage and sampling cone voltage were set to $3500 \mathrm{~V}$ and 80 $\mathrm{V}$, respectively.

\section{Results and Discussion}

3.1. Optimization of LC-MS/MS Conditions. In order to get best chromatographic resolution, many mobile phases including acetonitrile and methanol $0.05 \%, 0.1 \%$, and 0.2 $\%$ aqueous formic acid and acetic acid were tested in the present study. The best peak shape and resolution were obtained from a mixture of (A) water containing $0.1 \%$ formic acid and (B) acetonitrile containing $0.1 \%$ formic acid. The typical LC-MS/MS chromatograms of ginsenosides in standard mixtures, Shenfu Tang and Dushen Tang, are shown in Figure 2. From Figure 2, we can see that ginsenosides were separately eluted within $22 \mathrm{~min}$ by optimizing elution gradient. Meanwhile, ionization mode, capillary voltage, fragmentor voltage, collision energy, gas flow, and desolvation temperature were optimized as well.

The optimized MS/MS method could achieve highest response using the multiple reaction monitoring (MRM) pairs consisting of the precursor and product ions. IntelliStart (Waters Acquity UPLC system built-in software) was used to optimize the mass conditions for the ginsenosides. The results of IntelliStart for the 12 ginsenosides (including cone voltage optimization, optimized MS spectrum, collision energy optimization, and optimized daughter spectrum) can be founded in Supplementary Materials. The optimized precursor and product ions of the twelve ginsenosides are shown in Table 1. The optimum collision energy was determined to be from 36 $\mathrm{eV}$ to $64 \mathrm{eV}$ for different ginsenosides (Table 1).

3.2. Calibration Curves, LODs, and LOQs. The linearity of the developed method was assessed using six different concentrations of twelve ginsenosides. The standard calibration curves of all compounds were shown in Table 2 with satisfactory linearity $(r>0.9915)$. The LODs and LOQs of the twelve analytes were $0.30 \sim 8.00$ and $0.80 \sim 20.00 \mathrm{ng} / \mathrm{mL}$, respectively.

3.3. Precision, Repeatability, and Stability. The precision of the developed assay was evaluated with intraday and interday variations. The analyzed data showed that relative standard deviation (RSD) of intraday was in the range of 1.61-11.90\% at six times within 1 day, and the RSD of interday was in the range of $2.43-22.24 \%$ determined by repeated analysis of the sample for consecutive 3 days (Table 3 ). The repeatability was satisfactory with RSD below $9.58 \%$ by testing six independent samples (Table 3). For stability test, the samples were analyzed 


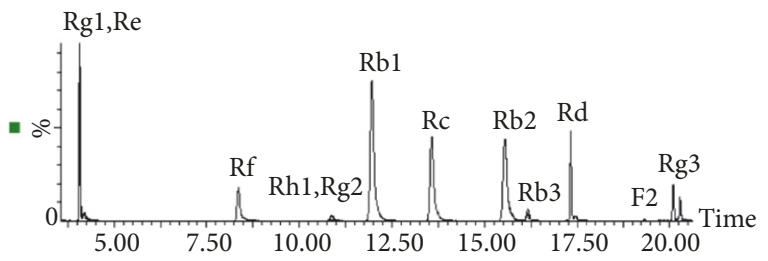

(a)

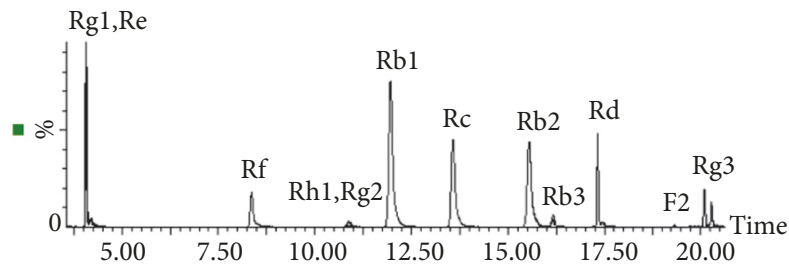

(b)

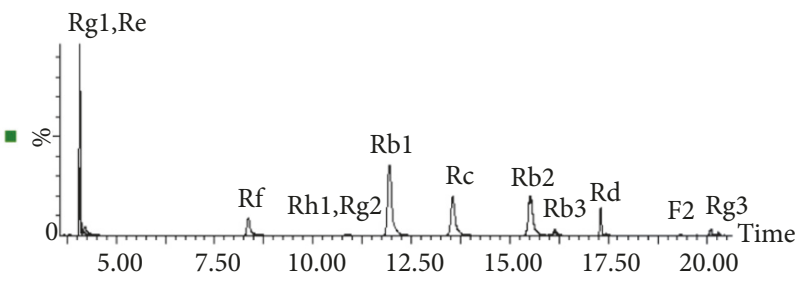

(c)

FIgURE 2: Typical UPLC-QQQ MS/MS chromatograms of twelve ginsenosides: (a) standard mixture, (b) Shenfu Tang, and (c) Dushen Tang.

TABLE 1: Mass spectra properties of twelve ginsenosides.

\begin{tabular}{|c|c|c|c|c|c|}
\hline Compounds & Precursor Ion & Product Ion & Dwell(s) & $\mathrm{CV}(\mathrm{V})$ & $\mathrm{CE}(\mathrm{V})$ \\
\hline Rh1 & 661.5 & 203.1 & 0.163 & 100 & 36 \\
\hline $\operatorname{Rg} 2$ & 807.7 & 349.2 & 0.163 & 100 & 44 \\
\hline Rg3 & 807.7 & 365.1 & 0.163 & 94 & 44 \\
\hline F2 & 807.7 & 627.5 & 0.163 & 28 & 40 \\
\hline $\mathrm{Rf}$ & 823.7 & 365.1 & 0.163 & 100 & 46 \\
\hline Rg1 & 823.7 & 643.5 & 0.330 & 100 & 38 \\
\hline $\mathrm{Re}$ & 969.8 & 789.6 & 0.163 & 100 & 42 \\
\hline $\mathrm{Rd}$ & 969.8 & 789.6 & 0.330 & 100 & 46 \\
\hline $\mathrm{Rc}$ & 1101.8 & 335.2 & 0.330 & 98 & 62 \\
\hline $\mathrm{Rb} 2$ & 1101.9 & 335.2 & 0.163 & 100 & 60 \\
\hline $\mathrm{Rb} 3$ & 1101.8 & 789.6 & 0.163 & 100 & 48 \\
\hline $\mathrm{Rb} 1$ & 1131.9 & 365.2 & 0.330 & 98 & 64 \\
\hline
\end{tabular}

TABLE 2: Calibration curves, LOD and LOQ for twelve ginsenosides.

\begin{tabular}{|c|c|c|c|c|c|}
\hline Compound Name & Calibration curve & $\mathrm{r}^{2}$ & $\begin{array}{c}\text { Linear range } \\
(\mathrm{ng} / \mathrm{mL})\end{array}$ & $\begin{array}{c}\text { LOD } \\
(\mathrm{ng} / \mathrm{mL})\end{array}$ & $\begin{array}{c}\text { LOQ } \\
(\mathrm{ng} / \mathrm{mL})\end{array}$ \\
\hline Rh1 & $\mathrm{Y}=1.2549 \mathrm{X}-0.1531$ & 0.9985 & $31.25-1000.00$ & 8.00 & 20.00 \\
\hline Rg2 & $Y=3.0121 X+4.5875$ & 0.9995 & $25.00-800.00$ & 4.00 & 12.00 \\
\hline Rg3 & $Y=5.7582 X+44.9854$ & 0.9993 & $62.50-2000.00$ & 0.30 & 0.80 \\
\hline $\mathrm{F} 2$ & $Y=19.1291 X+9.5143$ & 0.9948 & $1.09-35.00$ & 1.00 & 10.00 \\
\hline $\mathrm{Rf}$ & $\mathrm{Y}=13.2170 \mathrm{X}+30.8831$ & 0.9997 & $62.50-2000.00$ & 0.50 & 1.50 \\
\hline Rg1 & $Y=15.9033 X-11.0447$ & 0.9830 & $140.63-2250.00$ & 1.00 & 3.00 \\
\hline $\operatorname{Re}$ & $Y=3.1045 X+73.7435$ & 0.9981 & $140.63-4500.00$ & 0.60 & 1.50 \\
\hline $\mathrm{Rd}$ & $Y=9.4545 X+64.9408$ & 0.9974 & $46.88-1500.00$ & 0.60 & 2.50 \\
\hline $\mathrm{Rc}$ & $Y=18.2217 X-97.4775$ & 0.9993 & $78.13-2500.00$ & 0.50 & 2.40 \\
\hline $\mathrm{Rb} 2$ & $Y=18.0760 X-823.578$ & 0.9888 & $109.38-3500.00$ & 2.00 & 6.00 \\
\hline Rb3 & $Y=5.2107 X+16.0536$ & 0.9981 & $23.44-750.00$ & 3.50 & 10.00 \\
\hline Rb1 & $Y=20.6874 X-509.2980$ & 0.9987 & $109.38-3500.00$ & 0.80 & 2.40 \\
\hline
\end{tabular}


Table 3: Precision and Repeatability for the twelve ginsenosides.

\begin{tabular}{|c|c|c|c|c|c|c|c|}
\hline \multirow[t]{2}{*}{ Ginsenosides } & \multicolumn{3}{|c|}{$\begin{array}{c}\text { Interprecision RSD (\%) } \\
(\mathrm{n}=6)\end{array}$} & \multicolumn{3}{|c|}{$\begin{array}{c}\text { Intraprecision RSD (\%) } \\
(\mathrm{n}=3)\end{array}$} & \multirow{2}{*}{$\begin{array}{c}\text { Repeatability } \\
\text { RSD (\%) } \\
(n=6)\end{array}$} \\
\hline & $\mathrm{L}$ & $\mathrm{M}$ & $\mathrm{H}$ & $\mathrm{L}$ & M & $\mathrm{H}$ & \\
\hline$\overline{\mathrm{Rh} 1}$ & 8.59 & 10.33 & 2.00 & 16.62 & 9.88 & 10.36 & 9.16 \\
\hline Rg2 & 4.74 & 10.38 & 3.21 & 18.82 & 18.99 & 20.11 & 4.57 \\
\hline Rg3 & 2.94 & 3.33 & 2.12 & 5.03 & 8.81 & 6.00 & 3.76 \\
\hline $\mathrm{F} 2$ & 11.90 & 3.57 & 1.93 & 6.90 & 12.18 & 6.63 & 5.99 \\
\hline $\mathrm{Rf}$ & 2.70 & 4.31 & 2.75 & 22.24 & 19.71 & 15.89 & 3.04 \\
\hline Rg1 & 3.96 & 4.16 & 3.97 & 4.65 & 5.22 & 3.17 & 6.29 \\
\hline $\mathrm{Re}$ & 2.70 & 2.14 & 3.39 & 8.91 & 5.74 & 5.97 & 5.99 \\
\hline $\mathrm{Rd}$ & 2.70 & 2.26 & 3.18 & 8.58 & 5.51 & 4.92 & 5.98 \\
\hline $\mathrm{Rc}$ & 3.62 & 2.87 & 2.82 & 14.24 & 10.50 & 4.27 & 5.17 \\
\hline $\mathrm{Rb} 2$ & 3.86 & 3.33 & 1.78 & 14.79 & 10.66 & 2.43 & 3.91 \\
\hline $\mathrm{Rb} 3$ & 2.56 & 3.85 & 3.15 & 18.55 & 9.81 & 15.42 & 9.58 \\
\hline $\mathrm{Rb} 1$ & 3.49 & 3.01 & 1.61 & 14.51 & 9.42 & 10.41 & 4.55 \\
\hline
\end{tabular}

TABLE 4: Recovery for the twelve ginsenosides $(n=6)$.

\begin{tabular}{|c|c|c|c|c|c|}
\hline Compounds & $\begin{array}{c}\text { Initial amount } \\
\text { (ng) }\end{array}$ & $\begin{array}{l}\text { Added amount } \\
\text { (ng) }\end{array}$ & $\begin{array}{c}\text { Detected amount } \\
\text { (ng) }\end{array}$ & $\begin{array}{c}\text { Recovery } \\
(\%)\end{array}$ & $\begin{array}{l}\text { RSD } \\
(\%) \\
\end{array}$ \\
\hline Rhl & 109.04 & 110.00 & 210.53 & 92.26 & 5.40 \\
\hline Rg2 & 232.89 & 230.00 & 452.45 & 95.46 & 3.56 \\
\hline Rg3 & 401.41 & 410.00 & 840.59 & 107.12 & 1.65 \\
\hline $\mathrm{F} 2$ & 3.90 & 3.50 & 7.86 & 113.20 & 4.25 \\
\hline $\mathrm{Rf}$ & 264.02 & 270.00 & 528.47 & 97.95 & 3.03 \\
\hline Rg1 & 493.58 & 520.00 & 1064.54 & 109.80 & 3.62 \\
\hline $\mathrm{Re}$ & 1458.43 & 1550.00 & 3162.58 & 109.95 & 3.33 \\
\hline $\mathrm{Rd}$ & 479.99 & 510.00 & 1040.27 & 109.86 & 3.30 \\
\hline $\mathrm{Rc}$ & 684.71 & 710.00 & 1462.20 & 109.51 & 2.24 \\
\hline $\mathrm{Rb} 2$ & 769.51 & 790.00 & 1524.60 & 95.58 & 1.05 \\
\hline $\mathrm{Rb} 3$ & 152.45 & 150.00 & 296.00 & 95.70 & 2.45 \\
\hline $\mathrm{Rb} 1$ & 997.10 & 1000.00 & 2085.35 & 108.82 & 1.10 \\
\hline
\end{tabular}

at $3 \mathrm{~h}, 6 \mathrm{~h}, 9 \mathrm{~h}, 12 \mathrm{~h}$, and $24 \mathrm{~h}$ (Table S13). The RSD of stability test was in the range of $6.94-13.74 \%$.

3.4. Accuracy. The accuracy of the method was assessed by a recovery assay. Before we did the test of recovery, the initial amount of 12 ginsenosides were tested firstly in accordance with the methods mentioned above, and the spiked samples were then extracted, processed, and quantified. Table 4 showed that the recovery of the twelve ginsenosides ranged from $92.26 \%$ to $113.20 \%$, and their RSD values were all less than $5.4 \%$. Recovery data represented the accuracy of the method and is sufficient for further sample analysis.

3.5. Analysis of Ginsenosides in Dushen Tang and Shenfu Tang. The established analytical assay was subsequently applied for the simultaneous determination of the twelve ginsenosides in Dushen Tang and Shenfu Tang. From Figure 3, we can see that there are significant differences among the contents of the twelve ginsenosides between Dushen Tang and Shenfu Tang. The results showed most of ginsenosides; except Rg1 and Rb1 showed higher contents in Shenfu Tang compared to Dushen Tang according to crude botanicals per gram (Figure 3 ).

Composite formulae are one of the most important characteristics of TCM to obtain synergistic effects or to diminish possible adverse reactions. Usually, the therapeutic efficacy of herbal medicines is achieved by combinatorial components rather than single compound [26]. The curative effect of Shenfu Tang is an integrative result of a number of ginsenosides and alkaloids [19]. Therefore, the differences of twelve ginsenosides between Dushen Tang and Shenfu Tang could be explained by law of compatibility of medicines in Dushen Tang and Shenfu Tang. Further study for the changes of the active compounds in Dushen Tang and Shenfu Tang (that means before and after compatibility of ginseng) is needed to carry out.

\section{Conclusions}

In this study, a UPLC-MS/MS method for the simultaneous determination of twelve active ginsenosides in Dushen Tang 


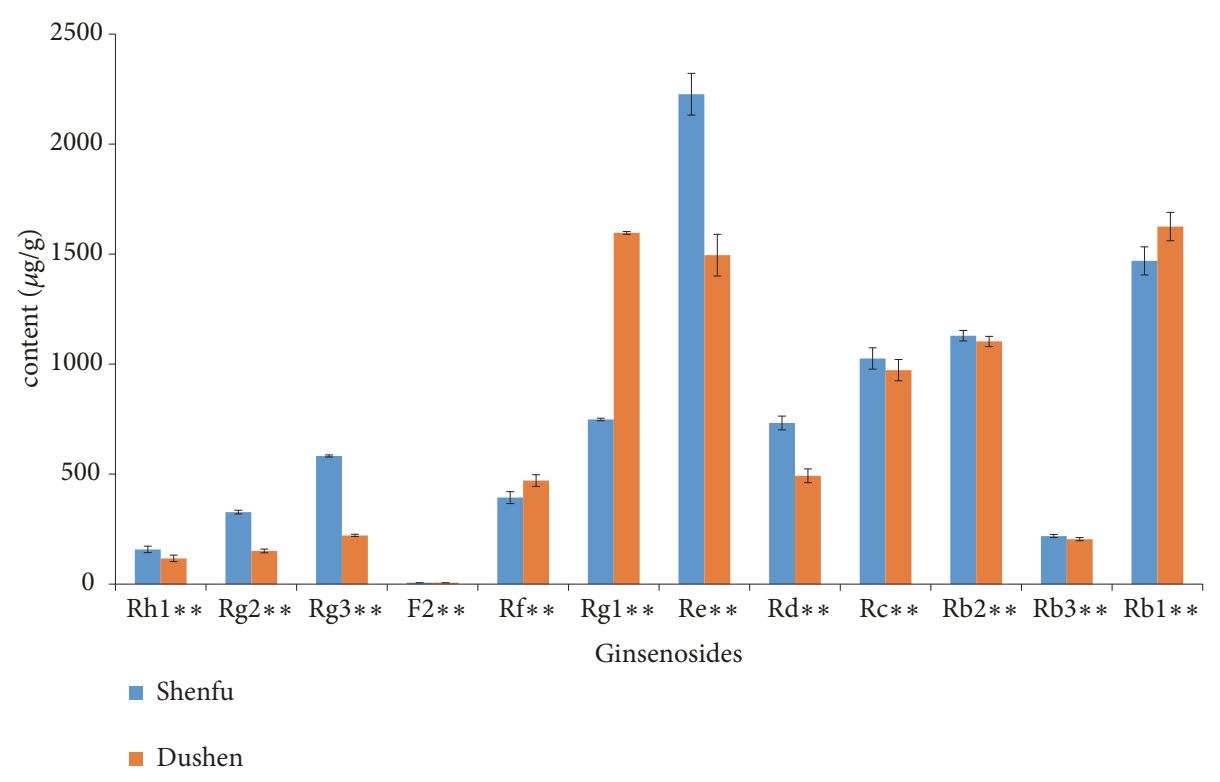

FIGURE 3: Concentrations of ginsenosides in Dushen Tang and Shenfu Tang. $* * \mathrm{p}<0.01$.

and Shenfu Tang has been developed. The results showed that it could be used for the quality control of Dushen Tang and Shenfu Tang. In addition, compared with Dushen Tang, most of ginsenosides except Rgl and Rb1 showed higher contents in Shenfu Tang; the different quantities of ginsenosides or active compounds in Dushen Tang and Shenfu Tang reflected the law of compatibility of medicines based on TCM theory.

\section{Data Availability}

The data used to support the findings of this study are available from the corresponding author upon request.

\section{Conflicts of Interest}

The authors declare that there are no conflicts of interest regarding the publication of this paper.

\section{Authors' Contributions}

Xiaofang Yang is the co-first author with Dawai Yang.

\section{Acknowledgments}

This research was supported by The Fundamental Research Funds for the Central Public Welfare Research Institutes (No. GH-2017-01-03, zz2017004), the National Natural Science Foundation of China (No. 81370095), National Science and Technology Major Project (No. 2017ZX09301040), and Beijing Key Laboratory of Research of Chinese Medicine on Prevention and Treatment for Major Diseases.

\section{Supplementary Materials}

Table S1. IntelliStart for Ginsenoside Rh1 Figure S1. A: Collision Energy Optimization $(\mathrm{m} / \mathrm{z}$ 661.50 $\longrightarrow 203.12)$; B: Optimized Daughter Spectrum (at collision Energy 36eV) Table S2. IntelliStart for Ginsenoside Rg2 Figure S2. A:
Collision Energy Optimization (m/z 807.66 $\longrightarrow 349.20)$; B: Optimized Daughter Spectrum (at collision Energy $44 \mathrm{eV}$ ) Table S3. IntelliStart for Ginsenoside Rg3 Figure S3. A: Collision Energy Optimization $(\mathrm{m} / \mathrm{z}$ 807.66 $\longrightarrow 365.13)$; B: Optimized Daughter Spectrum (at collision Energy 44eV) Table S4. IntelliStart for Ginsenoside F2 Figure S4. A: Collision Energy Optimization $(\mathrm{m} / \mathrm{z}$ 807.66 $\longrightarrow 627.54)$; B: Optimized Daughter Spectrum (at collision Energy 40eV) Table S5. IntelliStart for Ginsenoside Rf Figure S5. A: Collision Energy Optimization $(\mathrm{m} / \mathrm{z} 823.66 \longrightarrow 365.14)$; B: Optimized Daughter Spectrum (at collision Energy 46eV) Table S6. IntelliStart for Ginsenoside Rg1 Figure S6. A: Collision Energy Optimization $(\mathrm{m} / \mathrm{z}$ 823.66 $\longrightarrow 643.54)$; B: Optimized Daughter Spectrum (at collision Energy 38eV) Table S7. IntelliStart for Ginsenoside Re Figure S7. A: Collision Energy Optimization $(\mathrm{m} / \mathrm{z}$ 969.76 $\longrightarrow 789.60)$; B: Optimized Daughter Spectrum (at collision Energy $42 \mathrm{eV}$ ) Table S8. IntelliStart for Ginsenoside Rd Figure S8. A: Collision Energy Optimization $(\mathrm{m} / \mathrm{z}$ 969.76 $\longrightarrow 789.60)$; B: Optimized Daughter Spectrum (at collision Energy 46eV) Table S9. IntelliStart for Ginsenoside Rc Figure S9. A: Collision Energy Optimization $(\mathrm{m} / \mathrm{z}$ 1101.82 $\longrightarrow 335.15)$; B: Optimized Daughter Spectrum (at collision Energy 62eV) Table S10. IntelliStart for Ginsenoside Rb2 Figure S10. A: Collision Energy Optimization $(\mathrm{m} / \mathrm{z}$ 1101.89 $\longrightarrow 335.23)$; B: Optimized Daughter Spectrum (at collision Energy 60eV) Table S11. IntelliStart for Ginsenoside Rb3 Figure S11. A: Collision Energy Optimization (m/z 1101.82 $\longrightarrow 789.59) ;$ B: Optimized Daughter Spectrum (at collision Energy 48eV) Table S12. IntelliStart for Ginsenoside Rb1 Figure S12. A: Collision Energy Optimization $(\mathrm{m} / \mathrm{z}$ $1131.89 \longrightarrow 365.20$ ); B: Optimized Daughter Spectrum (at collision Energy 64eV) Table S13 Stability of twelve ginsenosides. (Supplementary Materials)

\section{References}

[1] D. Normile, "The new face of traditional Chinese medicine," Science, vol. 299, no. 5604, pp. 188-190, 2003. 
[2] J. L. Tang, B. Y. Liu, and K. W. Ma, "Traditional Chinese medicine," The Lancet, vol. 372, no. 9654, pp. 1938-1940, 2008.

[3] S. Li, S. Lai, J. Song et al., "Decocting-induced chemical transformations and global quality of Du-Shen-Tang, the decoction of ginseng evaluated by UPLC-Q-TOF-MS/MS based chemical profiling approach," Journal of Pharmaceutical and Biomedical Analysis, vol. 53, no. 4, pp. 946-957, 2010.

[4] J. Luo, S. Min, K. Wei, and J. Cao, "Ion channel mechanism and ingredient bases of Shenfu Decoction's cardiac electrophysiological effects," Journal of Ethnopharmacology, vol. 117, no. 3, pp. 439-445, 2008.

[5] D. Yang, X. Wang, Y. Wu et al., "Urinary metabolomic profiling reveals the effect of Shenfu decoction on chronic heart failure in rats," Molecules, vol. 20, no. 7, pp. 11915-11929, 2015.

[6] H. Wei, H. Wu, W. Yu, X. Yan, and X. Zhang, "Shenfu decoction as adjuvant therapy for improving quality of life and hepatic dysfunction in patients with symptomatic chronic heart failure," Journal of Ethnopharmacology, vol. 169, pp. 347-355, 2015.

[7] A. S. Attele, J. A. Wu, and C.-S. Yuan, "Ginseng pharmacology: multiple constituents and multiple actions," Biochemical Pharmacology, vol. 58, no. 11, pp. 1685-1693, 1999.

[8] C. Sun, Y. Li, Q. Wu et al., "De novo Sequencing and analysis of the American ginseng root transcriptome using a GS FLX Titanium platform to discover putative genes involved in ginsenoside biosynthesis," BMC Genomics, vol. 11, no. 1, p. 262, 2010.

[9] S.-Y. Nah, D.-H. Kim, and H. Rhim, "Ginsenosides: are any of them candidates for drugs acting on the central nervous system?” CNS Drug Reviews, vol. 13, no. 4, pp. 381-404, 2010.

[10] L. P. Christensen, "Ginsenosides: chemistry, biosynthesis, analysis, and potential health effects," Advances in Food and Nutrition Research, vol. 55, pp. 1-99, 2008.

[11] L. P. Christensen, M. Jensen, and U. Kidmose, "Simultaneous determination of ginsenosides and polyacetylenes in american ginseng root ( Panax quinquefolium L.) by high-performance liquid chromatography," Journal of Agricultural and Food Chemistry, vol. 54, no. 24, pp. 8995-9003, 2006.

[12] A. Kang, J. R. Guo, Z. Zhang, and X. L. Wang, "Simultaneous quantification of ten active components in traditional chinese formula sijunzi decoction using a UPLC-PDA method," Journal of Analytical Methods in Chemistry, vol. 2014, Article ID 570359, 8 pages, 2014.

[13] W. Zhou, J. Li, X. Li, Q. Yan, and P. Zhou, "Development and validation of a reversed-phase HPLC method for quantitative determination of ginsenosides $\mathrm{Rb} 1, \mathrm{Rd}, \mathrm{F} 2$, and compound $\mathrm{K}$ during the process of biotransformation of ginsenoside Rbl," Journal of Separation Science, vol. 31, no. 6-7, pp. 921-925, 2008.

[14] E. W. Chung, W. H. Kwok, D. K. Leung et al., "Detection and confirmation of ginsenosides in horse urine by GC-MS and LC-MS," Chromatographia, vol. 69, no. 9-10, pp. 923-932, 2009.

[15] J. Liu, Y. Liu, Y. Wang, A. Abozeid, Y. Zu, and Z. Tang, “The integration of GC-MS and LC-MS to assay the metabolomics profiling in Panax ginseng and Panax quinquefolius reveals a tissue- and species-specific connectivity of primary metabolites and ginsenosides accumulation," Journal of Pharmaceutical and Biomedical Analysis, vol. 135, pp. 176-185, 2017.

[16] T. Ligor, A. Ludwiczuk, T. Wolski, and B. Buszewski, "Isolation and determination of ginsenosides in American ginseng leaves and root extracts by LC-MS," Analytical and Bioanalytical Chemistry, vol. 383, no. 7-8, pp. 1098-1105, 2005.
[17] X. Ma, H. Xiao, and X. Liang, "Identification of ginsenosides in Panax quinquefolium by LC-MS," Chromatographia, vol. 64, no. 1-2, pp. 31-36, 2006.

[18] W. Wu, C. Jiao, H. Li, Y. Ma, L. Jiao, and S. Liu, " LC-MS based metabolic and metabonomic studies of Panax ginseng," Phytochemical Analysis, vol. 29, no. 4, pp. 331-340, 2018.

[19] N. Guo, M. Liu, D.-W. Yang et al., "Quantitative LC-MS/MS analysis of seven ginsenosides and three aconitum alkaloids in Shen-Fu decoction," Chemistry Central Journal, vol. 7, no. 1, p. 165, 2013.

[20] F. Liu, N. Ma, C. He et al., "Qualitative and quantitative analysis of the saponins in Panax notoginseng leaves using ultra-performance liquid chromatography coupled with timeof-flight tandem mass spectrometry and high performance liquid chromatography coupled with UV detector," Journal of Ginseng Research, vol. 42, no. 2, pp. 149-157, 2018.

[21] J. Chen, X. Guo, Y. Song, M. Zhao, P. Tu, and Y. Jiang, "MRM-based strategy for the homolog-focused detection of minor ginsenosides from notoginseng total saponins by ultraperformance liquid chromatography coupled with hybrid triple quadrupole-linear ion trap mass spectrometry," RSC Advances, vol. 6, no. 98, pp. 96376-96388, 2016.

[22] Y. Wang, J.-Y. Pan, X.-Y. Xiao, R.-C. Lin, and Y.-Y. Cheng, "Simultaneous determination of ginsenosides in Panax ginseng with different growth ages using high-performance liquid chromatography-mass spectrometry," Phytochemical Analysis, vol. 17, no. 6, pp. 424-430, 2006.

[23] H.-P. Wang, Y.-B. Zhang, X.-W. Yang, D.-Q. Zhao, and Y.-P. Wang, "Rapid characterization of ginsenosides in the roots and rhizomes of panax ginseng by UPLC-DAD-QTOF-MS/MS and simultaneous determination of 19 ginsenosides by HPLC-ESIMS," Journal of Ginseng Research, vol. 40, no. 4, pp. 382-394, 2016.

[24] W. Shi, Y. Wang, J. Li, H. Zhang, and L. Ding, "Investigation of ginsenosides in different parts and ages of Panax ginseng," Food Chemistry, vol. 102, no. 3, pp. 664-668, 2007.

[25] N. Guo, K. Ablajan, B. Fan, H. Yan, Y. Yu, and D. Dou, "Simultaneous determination of seven ginsenosides in Du Shen Tang decoction by rapid resolution liquid chromatography (RRLC) coupled with tandem mass spectrometry," Food Chemistry, vol. 141, no. 4, pp. 4046-4050, 2013.

[26] P. Liu, H. Yang, F. Long et al., "Bioactive equivalence of combinatorial components identified in screening of an herbal medicine," Pharmaceutical Research, vol. 31, no. 7, pp. 1788-1800, 2014. 

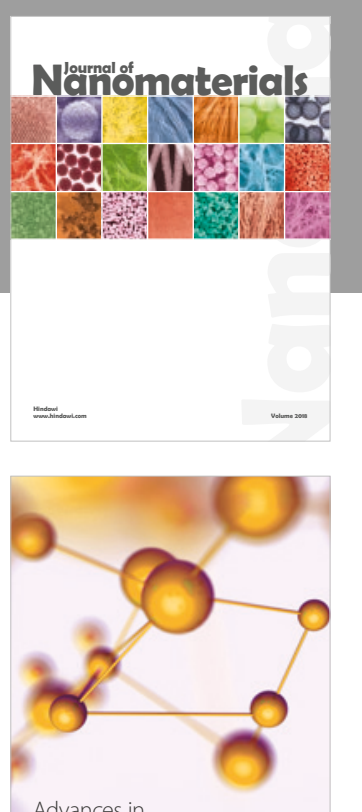

Physical Chemistry
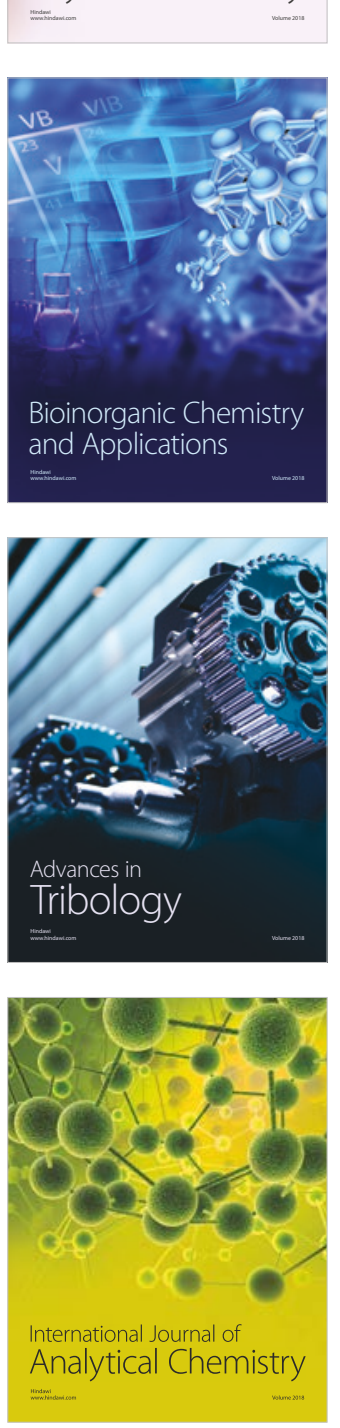

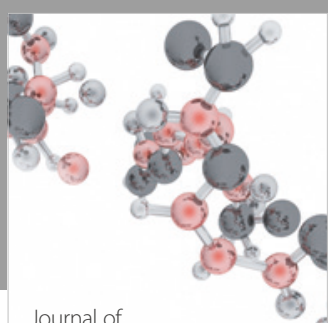

Analytical Methods

in Chemistry

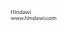

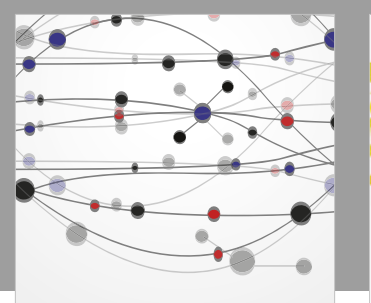

The Scientific World Journal

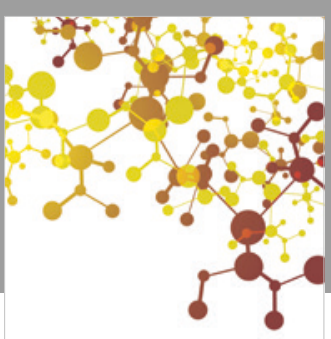

Journal of

Applied Chemistry
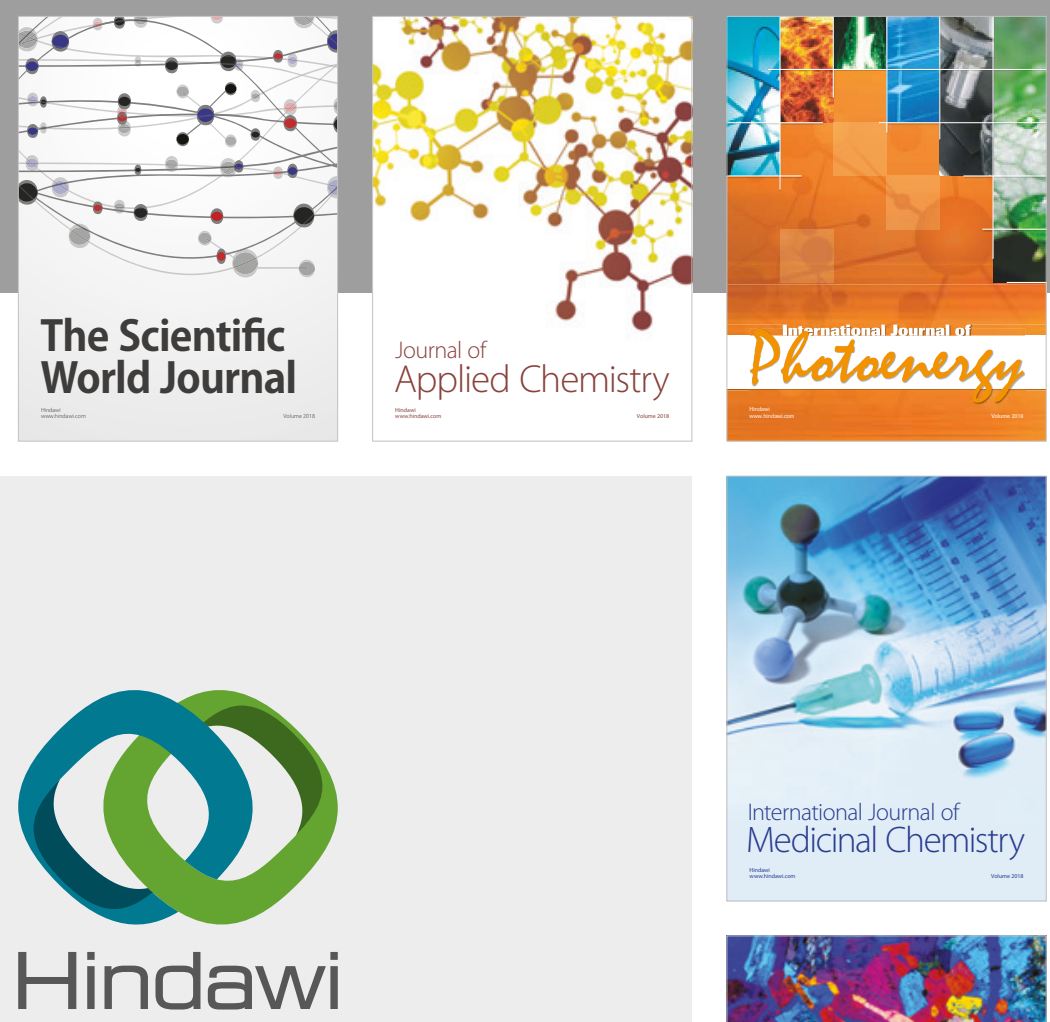

Submit your manuscripts at

www.hindawi.com
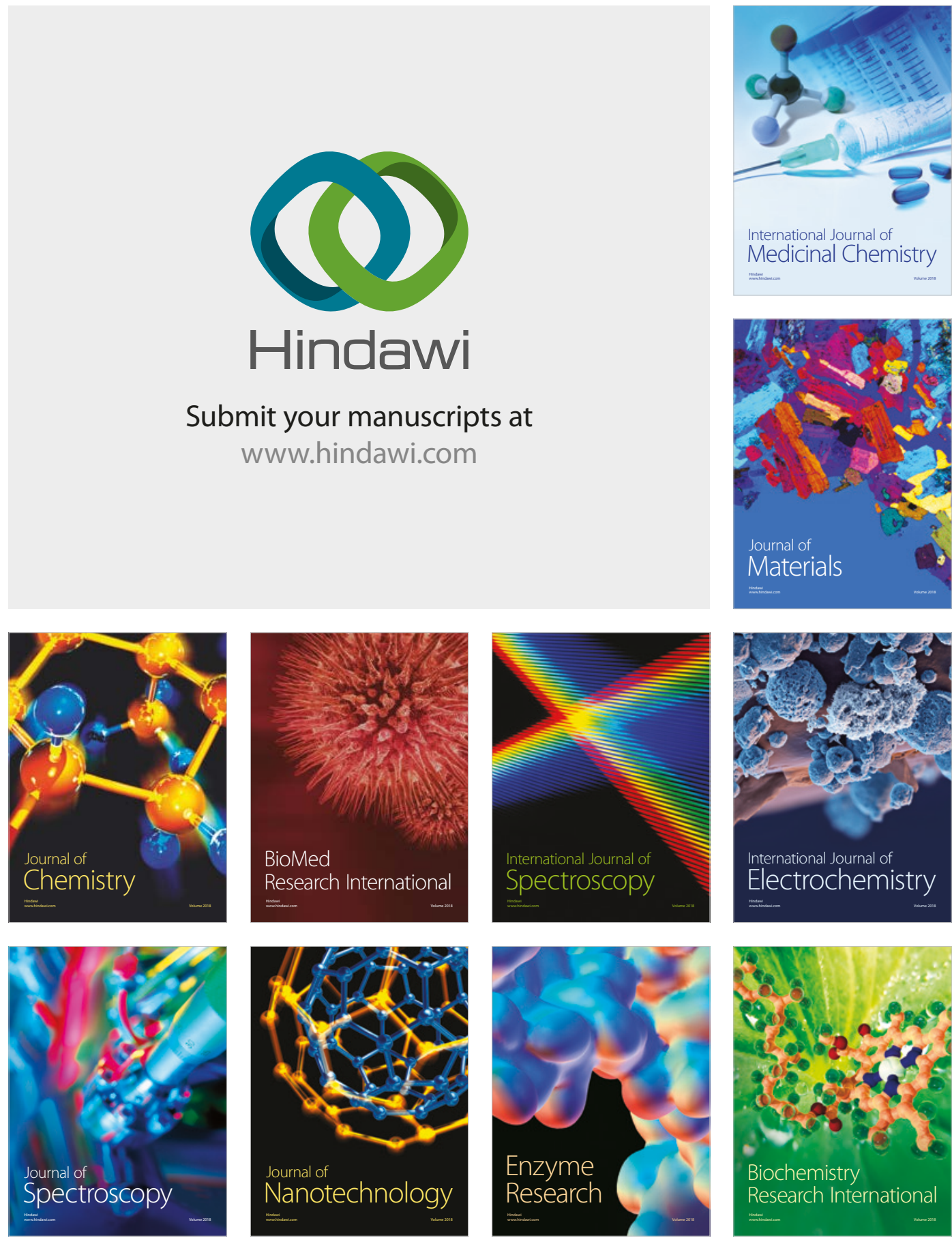
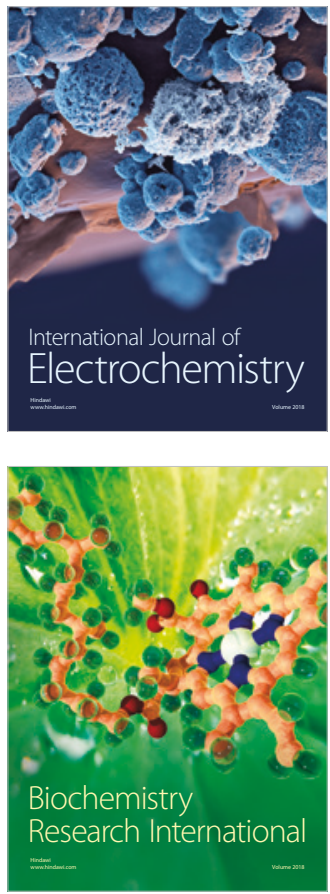